\title{
Utilizing Diffraction-Contrast Tomographic Reconstructions to Understand Hydride Volume Expansion Accommodation Processes in Zr Alloy.
}

\author{
C. D. Smith and I.M. Robertson \\ Dept. of Materials Science and Engineering, U. Illinois, 1304 W. Green Street, Urbana IL 61801.
}

In metal hydride forming systems such as $\mathrm{Ti}$ and $\mathrm{Zr}$, the volume expansion associated with a hydride is accommodated by elastic and plastic processes in the matrix and in some systems by internal twinning as well as the development of compositional gradients in the hydride that minimize the lattice mismatch at the hydride-matrix interface [1-2]. To develop physically-based models of the evolution of the hydride field and its impact on the mechanical properties, it is necessary to understand these volume change accommodation processes during multiple hydrogen chargingdischarging cycles. Models of the evolution of the hydride field have demonstrated it is essential to account properly for the volume accommodation mechanisms for a realistic distribution to be achieved [3].

To understand how dislocations are produced and distributed to accommodate the hydride volume expansion, we are coupling conventional imaging and analysis with tomographic reconstruction of hydrides formed in a commercial grade zirconium alloy. The tomographic reconstruction is made from a series of bright-field diffraction contrast images taken over a large angular range with images acquired every one degree. The reconstruction is enabled through use of EM3D 2.0 Software for Electron Microscope Tomography (McMahan Lab, Stanford University) for the alignment of the tiltseries data and the reconstruction of the 3D volume via filtered back-projection and UCSF Chimera 1.4 an Extensible Molecular Modeling System (University of California) for the visual interpretation of the reconstruction. To aid the interpretation of the tomographic images and to explore possible means of reducing the number of images needed in the reconstruction without loss of important information, we are exploring utilizing the fact dislocations terminate at other defects or junctions to outline the dislocations. The inverted contrast images shown in Figure 1 are select views of a zirconium hydride and the accommodation loops from the tomographic reconstruction and show the effect of using 78 images over an angular range of -45 to +33 degrees. The loss of information in the z-direction - the missing wedge effect - is obvious via elongation of features. The dislocations were delineated with the volume tracer tool in UCSF Chimera by maneuvering the lines drawn in the 3D model to the center of the region of highest contrast. Of course, this assumes this is an adequate representation of the location of the dislocation image under this diffraction condition - the validity of this assumption is currently being investigated by using different diffraction imaging conditions and acquiring images over a larger angular range. The final images show the traced objects superimposed on the reconstructed series. The superposition is good. Assuming this approach is reasonable for the intended purpose, these reconstructed images show the emitted dislocations are coplanar. The effectiveness of this dislocation field at accommodating the volume expansion will be compared to the lattice expansion associated with the hydride.

The authors gratefully acknowledge the Department of Energy NNSA program for support [4]. 


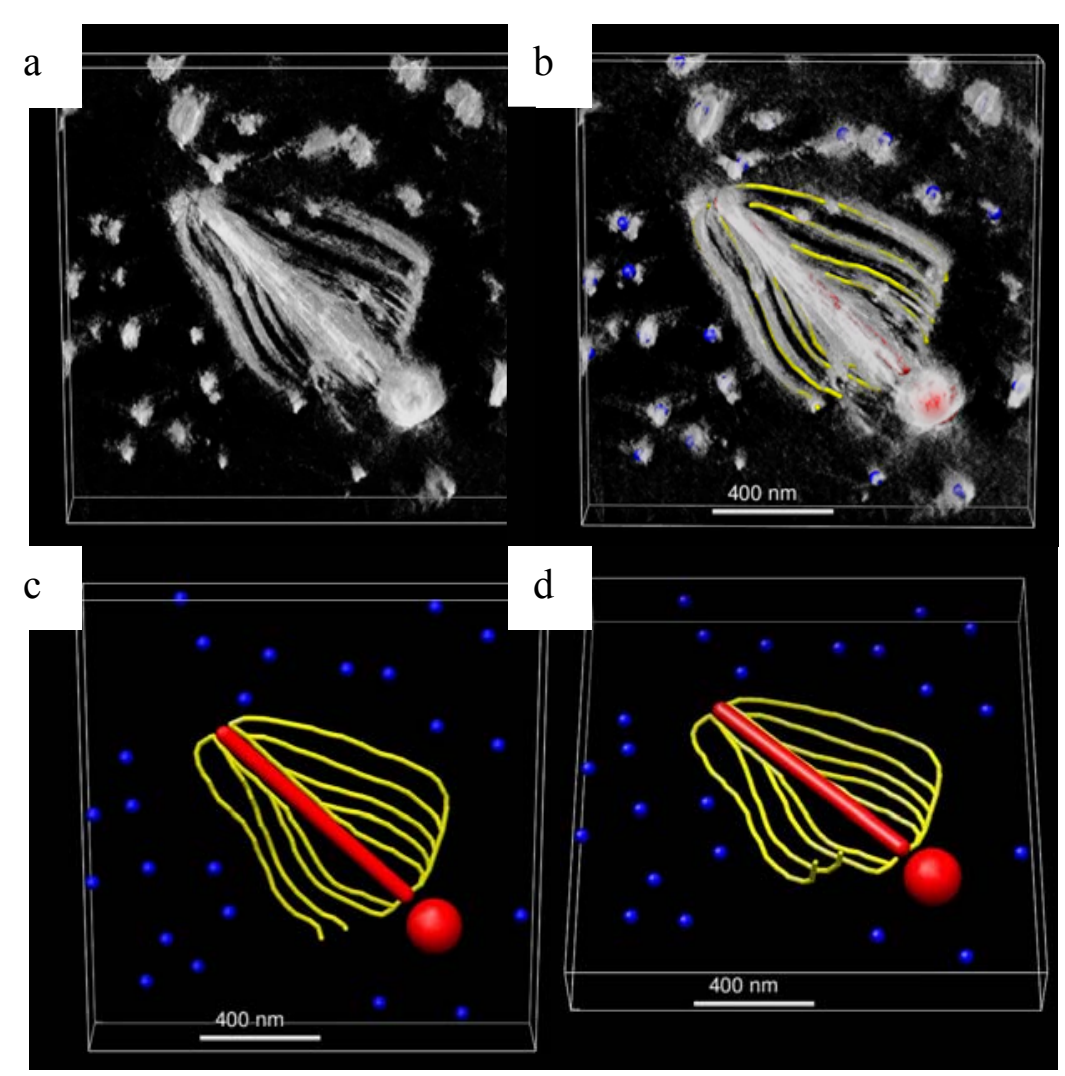

Fig. 1. a) The tomographic reconstruction of a large hydride with accommodation loops along with smaller hydrides surrounding it. b) Traced dislocations and hydrides overlaid with the reconstruction demonstrating the superposition. c) The traced dislocations (yellow) surrounding the large hydride (red) with the reconstruction contrast removed for clarity. d) Another view of the traced reconstruction, showing two of the dislocations terminating at the TEM thin foil surface. Note the contrast is inverted in images $a$ and $b$.

\section{REFERENCES}

[1] Xiao H. Effects of Hydrogen and Stress on the Microstructure and Microchemistry of $\mathrm{Ti}_{3} \mathrm{Al}$ Based Intermetallic Compounds. Material Science and Engineering. Urbana: University of Illinois, 1993.

[2] Shih DS, Robertson IM, Birnbaum HK. Hydrogen embrittlement of alpha titanium: in situ TEM studies. Acta Metall. 1988;36:111.

[3] Lufrano J. Sofronis P., Enhanced hydrogen concentrations ahead of rounded notches and cracks-competition between plastic strain and hydrostatic stress. Acta Mater. 1998;46:1519.

[4] Funding was provided through contract DEFG52-06-NA26150. The electron microscopy was performed in the Center for Microanalysis of Materials in the Frederick Seitz Materials Research Laboratory, University of Illinois 